\title{
NeuroImage
}

ELSEVIER

www.elsevier.com/locate/ynimg

NeuroImage 32 (2006) $62-69$

\section{In vivo micro-MRI of intracortical neurovasculature}

\author{
Patrick J. Bolan, ${ }^{*}$ Essa Yacoub, Michael Garwood, Kamil Ugurbil, and Noam Harel \\ Center for Magnetic Resonance Research, Department of Radiology, University of Minnesota School of Medicine, \\ Minneapolis, 2021 Sixth Street SE, Minneapolis, MN 55455, USA
}

Received 13 October 2005; revised 8 March 2006; accepted 14 March 2006

Available online 3 May 2006

\begin{abstract}
This work describes a methodology for in vivo MR imaging of arteries and veins within the visual cortex of the cat brain. Very high magnetic fields $(9.4 \mathrm{~T})$ and small field-of-view 3D acquisitions were used to image the neurovasculature at resolutions approaching the microscopic scale. A combination of time-of-flight $M R$ angiography and $T_{2}^{*}$ weighted imaging, using both endogenous BOLD contrast and an exogenous iron-oxide contrast agent, provided high specificity for distinguishing between arteries and veins within the cortex. These acquisition techniques, combined with 3D image processing and display methods, were used to detect and visualize intracortical arteries and veins with diameters smaller than $100 \mu \mathrm{m}$. This methodology can be used for visualizing the neurovasculature or building models of the vascular network and may benefit a variety of research applications including fMRI, cerebrovascular disease, and cancer angiogenesis.
\end{abstract}

(C) 2006 Elsevier Inc. All rights reserved.

\section{Introduction}

Methods for in vivo imaging of the cerebral vascular anatomy are important for a wide range of neuroscience applications, including studies of both normal physiology and disease states such as stroke and cancer. Magnetic resonance imaging offers a number of tools for directly and indirectly imaging the vasculature. Techniques such as arterial spin labeling and dynamic contrast-enhanced imaging can be used to characterize perfusion in capillaries and vessels smaller than the image voxel size. Direct imaging of distinct vessels, however, generally requires image resolution comparable to the vessel diameter. Several groups have shown very high-resolution $(\sim 100 \mu \mathrm{m})$ images of cerebral vasculature in animal models using magnetic resonance angiography, with either exogenous contrast agents (Mellin et al., 1994) or time-offlight angiography (Reese et al., 1999). Such methods have been used to study transient focal ischemia (Beckmann et al., 1999; Besselmann et al., 2001), genetic variation of vascular structure (Beckmann, 2000), and vascular abnormalities associated with Alzheimer's disease (Krucker et

\footnotetext{
* Corresponding author Fax: +1 612/626 2004.

E-mail address: bolan@cmrr.umn.edu (P.J. Bolan).

Available online on ScienceDirect (www.sciencedirect.com).
}

al., 2004). These studies have demonstrated that the major surface vessels of the brain can be readily imaged. Most of the intracortical arteries and veins, which branch off of the larger vessels and penetrate the cortex perpendicular to the pial surface, are too small to be seen in these MRA images.

Some of the larger intracortical veins, however, can be imaged using MR venograms or susceptibility-weighted images (Ogawa et al., 1990; Reichenbach et al., 1997). The principle intracortical veins, as described by Duvernoy et al. (1981) in ex vivo studies of human cadavers, penetrate the cortical surface at regular intervals and extend into the deep cortical layers and even into the underlying white matter. Due to their large diameter $(\sim 100 \mu \mathrm{m})$ and their elevated concentration of deoxyhemoglobin, these vessels cause magnetic field distortions and consequent signal dephasing in the cortical tissue adjacent to the veins. This blood oxygenation level-dependent (BOLD) effect enables these vessels to be seen on $\mathrm{T}_{2}^{*}$-weighted gradient echo acquisitions with image voxel sizes larger than the vessels themselves. Such images are now commonly used in animal fMRI studies to identify the vascular architecture (Pfeuffer et al., 2004; Park et al., 2005). However, this approach only allows visualization of the venous portion of the intracortical vasculature because it is specific to increased deoxyhemoglobin concentration.

In this work, we describe a combination of MR techniques that allows imaging of both arteries and veins within the cortex. Highresolution 3D gradient echo images were acquired in the cat visual cortex at a high magnetic field $(9.4 \mathrm{~T})$ and using a small surface coil for maximal sensitivity. The imaging methods included time-of-flight MR angiography and $\mathrm{T}_{2}^{*}$-weighted imaging with contrast from both endogenous BOLD effects and an exogenous contrast agent. The time-of-flight images were used to distinguish between arteries and veins; arteries appeared bright due to inflowing blood, and veins appeared dark due to the BOLD effect. $\mathrm{T}_{2}^{*}$-weighted images acquired with MION contrast agent gave high sensitivity for detecting both arteries and veins, but showed an exaggerated vessel diameter due to extravascular dephasing effects. These techniques, combined with various image reconstruction methods, enable in vivo visualization of numerous intracortical arteries and veins at microscopic resolution. This work demonstrates the feasibility of these techniques and lays the groundwork for further development in quantitative imaging and modeling of microvasculature. 


\section{Materials and methods}

Animal procedures were approved by our institution's animal care and use committee. Seven female cats $(\sim 1.4 \mathrm{~kg}, 13$ weeks old) were prepared as described previously (Harel et al., 2006). Briefly, the animals were initially anesthetized with an intramuscular injection of a ketamine $(10-25 \mathrm{mg} / \mathrm{kg})$ and xylazine $(2.5 \mathrm{mg} / \mathrm{kg})$ cocktail. They were then orally intubated and kept under isoflurane anesthesia ( $1 \%$ in a 7:3 $\mathrm{N}_{2} \mathrm{O}: \mathrm{O}_{2}$ mixture) for the entire experiment. An arterial line was placed in the femoral artery for monitoring blood pressure, and a venous line was placed in the femoral vein for administration of fluids and exogenous contrast agents. Normal body temperature $\left(38.5^{\circ} \mathrm{C}\right)$ was maintained using a water bath and feedback from a rectal thermometer. End-tidal $p \mathrm{CO}_{2}$ was continuously monitored and ventilator was adjusted to maintain the pressure between $32-35 \mathrm{~mm} \mathrm{Hg}$. Under these conditions, arterial blood pressure was generally in the range of $80-100 \mathrm{~mm} \mathrm{Hg}$. After preparation, cats were positioned in a holder using mouth and ear bars to minimize motion. A 14-mm diameter surface coil was positioned over the posterior portion of the animal's skull.

MR imaging was performed using a 9.4-T, 31-cm bore horizontal magnet (Magnex Scientific, Abingdon, UK) interfaced with a Varian console (Varian, Palo Alto, CA). The system was equipped with an actively shielded gradient coil insert (Magnex Scientific) with an inner diameter of $11 \mathrm{~cm}$, capable of a maximum gradient strength of $300 \mathrm{mT} / \mathrm{m}$ with a $300-\mu$ s rise time. After animal preparation, the holder was inserted into the scanner and scout images were acquired to verify correct positioning. The $\mathrm{B}_{0}$ homogeneity was maximized over a $10 \times 10 \times 10 \mathrm{~mm}$ region of interest in the visual cortex using FASTESTMAP (Gruetter, 1993). Low-resolution 3D gradient echo scout images were then acquired to allow precise slice positioning. High-resolution, 3D gradient echo images were acquired with typical values of FOV $20 \times 20 \times 5 \mathrm{~mm}$, matrix $256 \times 256 \times 32$, nominal resolution $78 \times 78 \times 156 \mu \mathrm{m}, \mathrm{TR} / \mathrm{TE}=32 / 5.5 \mathrm{~ms}$, readout band width $20 \mathrm{kHz}, \mathrm{NEX}=8$, total scan time $\sim 35 \mathrm{~min}$. No physiological triggering or gating was used. An asymmetric echo was used in the readout direction to reduce the echo time and gradient duty cycle (the percentage of $\mathrm{k}$-space before/after the echo was $37.5 / 62.5 \%$, compared with $50 / 50 \%$ for a symmetric echo). For flow-weighted images, a transverse slab (thickness $2 \mathrm{~mm}$ ) was positioned in the center of the cortex so that the superior edge of the slice passed through the sagittal sinus. The vertical position of the slice was critical for producing proper flow weighting in the intracortical arteries running perpendicular to the cortical surface. The excitation flip angle (nominally $30^{\circ}$ ) was adjusted experimentally to minimize the cortical tissue signal and maximize the signal from inflowing vessels. Precontrast tissue-weighted images were acquired using the same geometry and sequence parameters, but with a reduced flip angle (nominally $10^{\circ}$ ) adjusted to maximize cortical signal. Animals were then injected with a bolus of monocrystalline iron oxide contrast agent (MION) (Shen et al., 1993) at a dose of 7-10 mg $\mathrm{Fe} / \mathrm{kg}$. Postcontrast images were acquired using the same parameters as the precontrast tissue-weighted images.

Images were reconstructed using Matlab (Mathworks, Natick, MA). The asymmetric k-space was multiplied by a symmetric Tukey window function (Harris, 1978), with transition bands covering $20 \%$ of the k-space width on each edge. Conventional partial Fourier methods to symmetrize the k-space data were not used due to the potential for introducing additional artifacts (Liang et al., 1992). After filtering, $\mathrm{k}$-space was zero-filled to twice the acquisition matrix size and Fourier transformed. Image analysis, volume rendering, and manual segmentation were performed using the visualization package Amira (Mercury Computer Systems, Chelmsford, MA). Adobe Illustrator and Photoshop (Adobe Systems Inc., San Jose, CA) were used to create and annotate layered images for the 2D vessel mapping. Seven cats were imaged and visualized over the course of this study; a detailed quantitative image analysis is presented for only one of the seven data sets to demonstrate feasibility.

\section{Results}

\section{Time-of-flight imaging}

The technique of 3D time-of-flight microangiography is demonstrated in Fig. 1. The 2-mm axial excitation slab was positioned so that the superior edge of the slab passed through the central sagittal sinus, as can be seen in the coronal image (Fig. 1a). The flip angle $\left(30^{\circ}\right)$ is greater than the Ernst angle for both blood

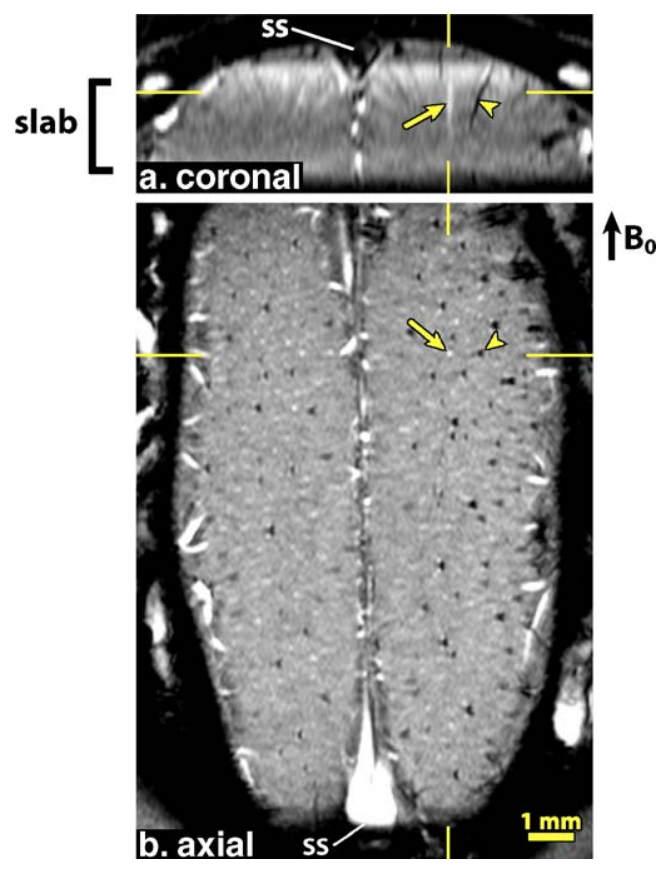

Fig. 1. An example of 3D time-of-flight microangiography in the cat visual cortex. Two orthogonal slices were extracted from a slab-selective, $\mathrm{T}_{1^{-}}$ weighted 3D gradient echo acquisition. The axial excitation slab (windowed sinc pulse, $2 \mathrm{~mm}$ thick, $30^{\circ} \mathrm{flip}$ ) was positioned so that its superior edge passed through the sagittal sinus (SS), as can be seen in the coronal (top) view. Arteries, indicated by arrows, are bright due to unsaturated blood flowing into the slab. Veins, indicated by arrowheads, are dark due to their elevated concentration of deoxyhemoglobin (the BOLD effect). In the axial (dorsal) view, most intracortical arteries and veins are oriented perpendicular to the imaging plane and appear circular or elliptical. The acquisition parameters included $\mathrm{TR} / \mathrm{TE}=32.4 / 5.5 \mathrm{~ms}$, readout band width $20 \mathrm{kHz}$, asymmetric echo in the readout direction $(37.5 / 62.5 \%)$, FOV $20 \times 20 \times 5$ mm, matrix $256 \times 256 \times 32,8$ averages, acquisition time 4:25 min per average, total time $35: 20 \mathrm{~min}$. The image was reconstructed by windowing with a symmetric Tukey window affecting $20 \%$ of pixels on each edge of $\mathrm{k}$ space, zero-filling to twice the native acquisition matrix and Fourier transforming, producing a reconstructed resolution of $39 \times 39 \times 78 \mu \mathrm{m}$. No partial Fourier conjugation or phase correction was used. Note that the sagittal sinusis bright in the axial image (b) because the flow is perpendicular to the excitation slab, whereas in the coronal image (a) the flow is parallel to the slab. 
$\left(\mathrm{T}_{1} \sim 2.4 \mathrm{~s}, \theta_{\text {ernst }}=9^{\circ}\right)$ (Dobre et al., 2005) and cortical grey matter (measured $\mathrm{T}_{1}=1.8 \mathrm{~s}, \theta_{\text {ernst }}=11^{\circ}$ ), so the spins inside the slice are saturated and show low signal. The two bright bands visible in the coronal (top) image correspond with the edges of the slab profile, where the effective flip angle is lower and the degree of saturation is reduced. Blood that flows into the slice is not saturated and therefore gives a higher signal than blood that has remained inside the slice for one or more excitations. This effect is called the timeof-flight or inflow effect. In Fig. 1, the yellow arrows show an example of a bright vessel in both views. Other bright vessels are evident in each view, particularly in the axial view in which most of the larger intracortical vessels are oriented perpendicular to the image plane. Note that these bright signals originate from water protons inside the vessel lumens, so the actual vessel diameters are comparable in size to these bright features.

Also evident in these images are intracortical veins, which appear dark due to their reduced level of oxygenation relative to arteries and tissue. This BOLD effect causes extravascular magnetic field gradients leading to spin dephasing (Ogawa et al., 1990). Inside blood vessels, magnetic field inhomogeneities exist around red blood cells; diffusion of blood spins in the presence of these inhomogeneities and exchange both in and around red blood cells leads to a steeply field-dependent decrease in apparent $T_{2}$. The consequence of these effects is signal loss in and around deoxyhemoglobin-containing blood vessels. For simplicity, we will refer to the BOLD effect as the cumulative consequence of the two effects, that is, dephasing due the extravascular field gradients and intravascular shortening of $\mathrm{T}_{2}$. At this high magnetic field, and with most veins in the imaging volume oriented approximately perpendicular to the external $\mathrm{B}_{0}$ field, the intracortical veins appear dark even at a relatively short echo time $(\mathrm{TE}=5.5 \mathrm{~ms})$. Numerous veins are visible in both views of Fig. 1. Note that the dark structures visible in these images have larger diameters than the vessel lumens themselves because they include extravascular dephasing effects.

Considering both the BOLD and inflow effects, we propose that the bright intracortical vessels are arteries whereas the dark intracortical vessels are veins. The intravascular signal is determined by both BOLD and flow effects, whereas the extravascular signal is only affected by BOLD and not by flow. Arterial blood has a magnetic susceptibility comparable to the surrounding cortical tissue, and thus these vessels display minimal BOLD effects. The inflow of relaxed arterial blood causes the arteries to appear bright. For veins, however, the BOLD signal decrease dominates over inflow effects, and the vessels appear as dark structures.

\section{Contrast-enhanced imaging}

Using time-of flight imaging to visualize arteries has limited applicability because the inflow effect is only clear near the edge of the excitation slab. The arterial visibility can be improved by injecting an intravascular iron-oxide-based contrast agent (MION). MION is superparamagnetic, has a very high $R_{2}$ relaxivity $\left(R_{2}=\right.$ $58.79 \mathrm{~s}^{-1}$ ), remains in the intravascular space (particle size $22 \mathrm{~nm}$ ), and has a long blood half-life (Shen et al., 1993). When used in high concentrations, as in this study, the increased intravascular $\mathrm{T}_{2}$ relaxation dominates over flow effects, causing both arteries and veins to appear dark in gradient echo images. The combined use of both inflow weighting and exogenous contrast imaging is demonstrated in the series of images shown in Fig. 2. Fig. 2a shows an axial slice from a $3 \mathrm{D}$ flow-weighted image using the same parameters as in Fig. 1. Here, the intracortical arteries appear bright and the veins appear dark. A second scan (Fig. 2b) was acquired with a reduced flip angle to increase the signal from the cortical tissue. Note that in this tissue-weighted scan, the flow effects are no longer visible and the arteries are indistinguishable from the surrounding cortex.

Fig. 2c shows an image acquired with the same parameters as the image in Fig. $2 \mathrm{~b}$ but after the injection of MION. In the postMION image, both arteries and veins appear as dark structures. As with the pre-MION BOLD effect in veins, the apparent size of each vessel, both arterial and venous, is larger in the post-MION image than the actual vessel lumen due to the susceptibility-induced extravascular dephasing. A large number of small vessels that were not visible in the pre-MION or flow-weighted images can now be detected in this post-MION image. It is not known if these small
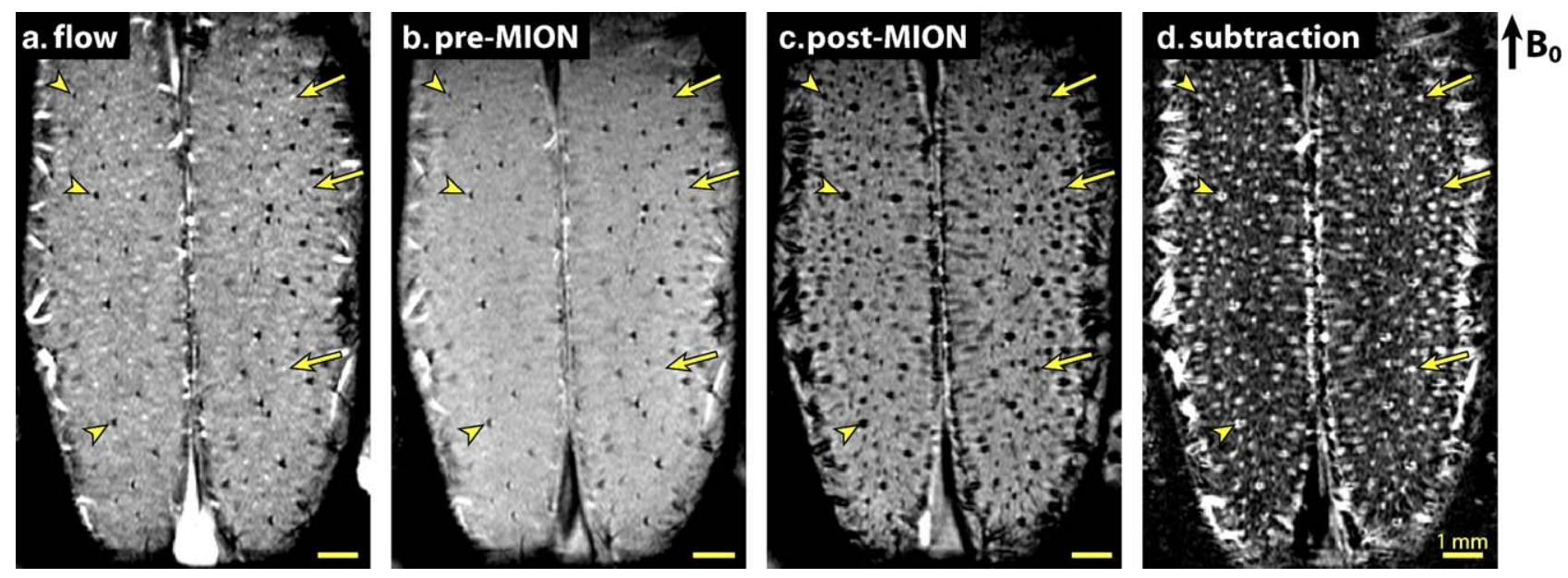

Fig. 2. A series of three images acquired with (a) flow weighting, (b) tissue weighting without exogenous contrast, and (c) tissue weighting with MION. A subtraction image (d) was produced by subtracting the post-MION image from the pre-MION image. Arteries (arrows) are bright in the flow-weighted image, undetectable in the tissue-weighted image, dark in the post-MION image, and bright in the subtraction. Veins (arrowheads) are dark in both pre-MION images, dark and larger in the post-MION image, and bright in the subtraction. The larger veins have a ring-like appearance in the subtraction image because they were dark prior to the MION administration. All images $(\mathrm{a}-\mathrm{c})$ used the same acquisition and reconstruction methods as in Fig. 1, except that for the tissue-weighted images panels $\mathrm{b}$ and $\mathrm{c}$, the flip angle was reduced from $30^{\circ}$ to $10^{\circ}$. The parameters and grayscale windowing are identical for panels $\mathrm{b}$ and $\mathrm{c}$. 
vessels are arteries or veins because all vessels are dark after MION injection. Note also the general darkening of the signal from the cortical tissue relative to Fig. $2 b$, which contains numerous small vessels and capillaries that cannot be distinguished at this resolution.

By subtracting the post-MION image from the pre-MION image, a subtraction image can be made, which shows positive signal in pixels where the MION caused a signal loss due to increased signal dephasing (Fig. 2d). This image can be used like a conventional angiographic data set for creating maximum intensity projections (MIPs) or other volume-rendered visualizations. Note that several of the larger veins have a ring shape, as there was little signal in the center of these vessels prior to MION due to the BOLD effect.

\section{Distinguishing arteries and veins}

The series of three images shown in Figs. $2 \mathrm{a}-\mathrm{c}$ represent the proposed imaging methodology in this work. The post-MION image has the greatest sensitivity for detecting small vessels of both types, whereas the flow-weighted image and pre-MION tissue-weighted image can be compared to identify whether a vessel is an artery or vein. By carefully comparing all three images, a large number of vessels can be identified in a single slice, as shown in Fig. 3a. This two-dimensional vessel map was created by overlaying the images from Figs. 2a to $\mathrm{c}$ and annotating them using Adobe Illustrator. First, vessels were identified in the flowweighted image (Fig. 2a): bright vessels were tentatively labeled arteries, and dark vessels were labeled veins. Each vessel was then checked in the pre-MION image (Fig. 2b) to verify that arteries were not detectable and veins were dark. Then the post-MION image (Fig. 2c) was evaluated to verify that both arteries and veins had distinct, dark outlines that did not overlap with adjacent vessels. In most cases, the vessel outline could be approximated with either a circle or an ellipse. Vessels that did not have consistent geometry in all three views were removed from the analysis. For example, there were approximately 440 dark, ellipsoidal structures visible in Fig. 2c. In comparing each of these with the pre-MION and flow-weighted images, it was found that 287 of these $(65 \%)$ were either not consistently visible or were not clearly identifiable as either an artery or a vein. For each of the remaining 152 vessels, an ellipse was overlaid on the post-MION image (Fig. 2c) and sized to completely cover the dark region associated with the vessel. The vessel ellipses were drawn on the post-MION image because this image offered the most distinct view of the vessel features. The sizes of the ellipses are somewhat arbitrary because they depend on the grayscale windowing used for the image, but they generally represent the extent of extravascular dephasing for both arteries and veins.

The set of these ellipses, shown in Fig. 3a overlaid on the flowweighted image, represent a rudimentary model of the intracortical vascular structure in this $2 \mathrm{D}$ slice. This model shows the spatial distribution of the larger vessels, but it does not show the true vessel diameters; the extent of each ellipse can be considered an upper limit of the vessel diameter. Nevertheless, several observations can be made directly from these data. Firstly, the dephased regions associated with veins, with a mean minor diameter of $229 \mu \mathrm{m}$, is larger than those associated with arteries, which had a mean minor diameter of $142 \mu \mathrm{m}$ (note that these values were measured in a single animal). Two factors contribute to this: It is known that the intracortical veins typically have larger diameters than intracortical arteries (Duvernoy et al., 1981), and they also have more deoxyhemoglobin, which may add to the effect of MION to increase the size of the dephased extravascular region. Secondly, it can be seen that the distribution of arteries and veins is consistent with previous ex vivo studies of cortical vasculature. The largest veins oriented perpendicular to the image plane (and the cortical surface) are the principle intracortical veins. These are spaced $1-1.5 \mathrm{~mm}$
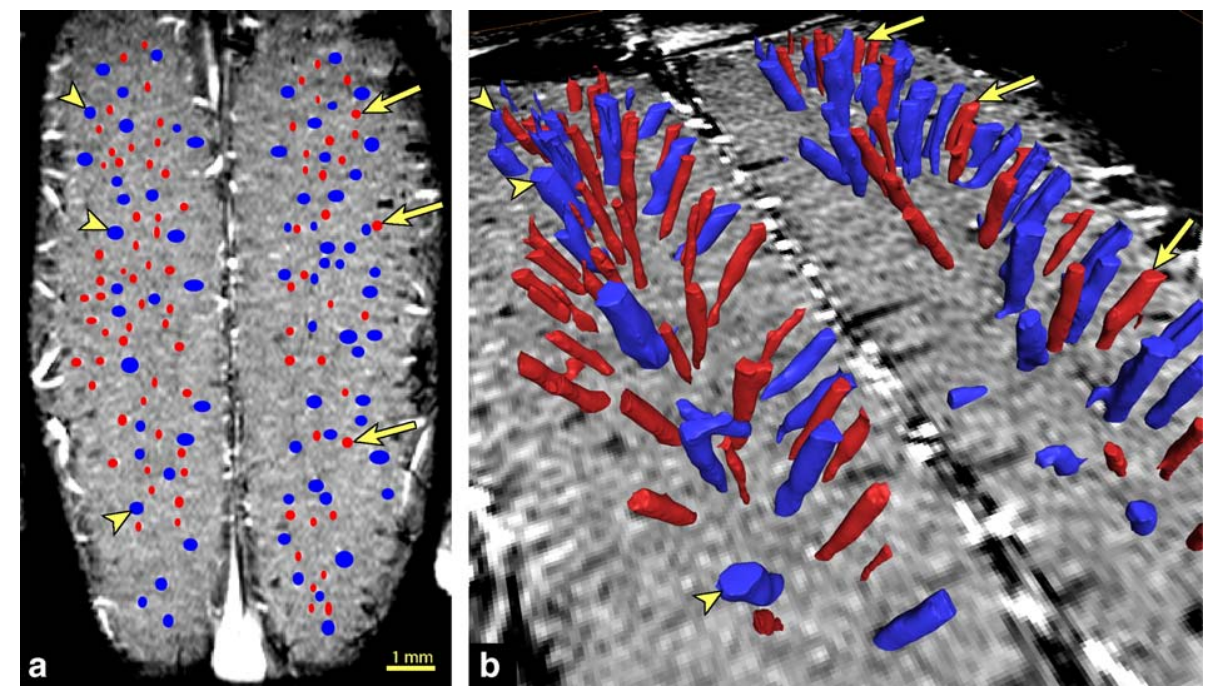

Fig. 3. Identification and reconstruction of arteries (red, arrows) and veins (blue, arrowheads) using the same data set as in Fig. 2. (a) Two-dimensional map of intracortical vessels, produced by placing ellipses over arteries and veins, and sizing the ellipses to cover the extent of the dark region of the post-MION image. The vessels identified in this specific slice from one animal include 83 arteries (with associated dephased regions having minor diameters ranging from 83 to $259 \mu \mathrm{m}$, mean $142 \mu \mathrm{m}$ ) and 69 veins (dephased region minor diameter 136-343 $\mu \mathrm{m}$, mean $229 \mu \mathrm{m}$ ). These measurements represent the extent of extravascular dephasing and are therefore larger than the vessels themselves. (b) Three-dimensional surface rendering of the vessels identified in panel a. For this reconstruction, the vessel extents were determined using the 3D subtraction volume. See the text for details of the reconstruction methods. Note that although these vessels have a variable angle of incidence with the imaging plane (within approximately $\pm 45^{\circ}$ ), all of these vessels are within $\sim 15^{\circ}$ of being perpendicular to $\mathrm{B}_{0}$. 
apart and are surrounded by a network of smaller veins and arteries oriented in the same direction.

Creating the two-dimensional vessel map of Fig 3a was a manually intensive task. Although this specific procedure would not be feasible for mapping the vasculature throughout the full imaging volume, a partially automated approach can be used to extend the segmentation into three dimensions. Fig. $3 \mathrm{~b}$ is an example of a three-dimensional surface rendering of the same vessels shown in Fig. 3a. Rather than repeating the 2D analysis on all image slices, this model was produced using the subtraction data shown in Fig. 2d. The subtracted volume image was normalized by dividing by the pre-MION image to correct for coil inhomogeneity, and then smoothed with a 3D Gaussian filter. The resulting volume was thresholded to produce a mask of all voxels that decreased in intensity after MION injection. The mask was then manually trimmed to include only those vessels chosen in Fig $3 \mathrm{a}$ and to remove those portions of the mask where the vessel extents were not clearly identifiable. Although this procedure also required substantial manual interaction, it could be further automated using vessel tracking or other computer vision algorithms.

With the full three-dimensional information, the extent of each vessel's penetration into the cortex can be determined. Vessels can then be classified using the method of Duvernoy et al. (1981), as done previously for venographic images (Park et al., 2005). It appears that the majority of the arteries visible in these images are of classes A4, A5, and A6, meaning they extend into the deep cortical layers (A4) or continue into the underlying white matter (A5 and A6). The veins also vary in their penetration and fall into the classes V4 and V5 of Duvernoy et al. The smallest vessels that are reliably detectable with this imaging method, the A4 arteries, are reported to have diameters of $30-40 \mu \mathrm{m}$ in human subjects (Duvernoy et al., 1981). Smaller vessels (A1-3, V1-3) are visible in the post-MION images but are not clearly identifiable in the flow-weighted or pre-MION images. It is not known, however, if intracortical vessels have similar diameters in both humans and cats, so the actual vessel sizes in this study are not known.

\section{Visualizations}

Figs. 4 and 5 give other examples of reconstruction methods used to visualize the vascular network. An example of a maximum intensity projection (MIP), commonly used for visualizing MR angiographic data, is shown in Fig. 4. These projections were created directly from the volumetric subtraction image with no manual modifications. These images, particularly the coronal view, give a good overview of the intracortical vascular architecture. Most distinctive are the large vessels that pass through the cortex nearly perpendicular to the pial surface, and then bend as they continue into the underlying white matter.

The post-MION images can also be reconstructed using methods commonly used for venograms, or susceptibility-weighted images (Reichenbach et al., 1997). Fig. 5a shows a single slice from a high-resolution coronal $3 \mathrm{D}$ data set (reconstructed resolution $26 \times 26 \times 78 \mu \mathrm{m})$. This image shows not only the principle intracortical veins (Duvernoy's V5), but also smaller penetrating vessels and some of the deep branches of the larger veins. The left side of Fig. $5 b$ shows a minimum intensity projection (MinIP) centered around the slice shown above. This reconstruction shows more vessels than the single slice because it includes data from a thicker slab $(515 \mu \mathrm{m})$. It may be possible to further improve the quality of vessel visualization by enhancing the
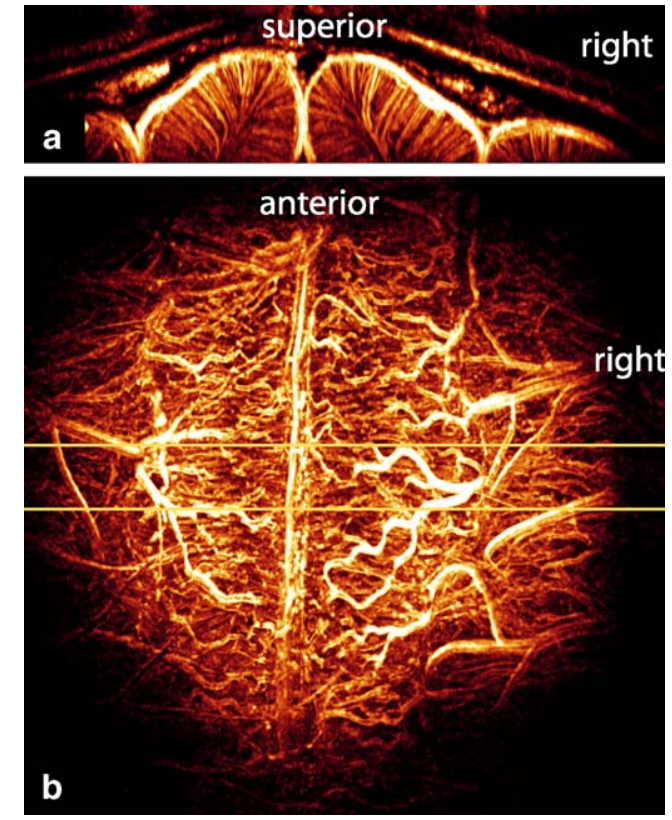

Fig. 4. Maximum intensity projections (MIPs) of the 3D subtraction image from Fig. 2d, viewed in (a) coronal and (b) axial orientations. The axial projection (b) was performed over the full acquisition volume, whereas the coronal projection (a) was produced from a narrow slab indicated by the yellow lines in panel $b$. The coronal view clearly shows the uniform organization of intracortical vessels. The axial view is dominated by the vessels on the pial surface. This figure demonstrates how the MION subtraction image can be used as a conventional MRA data set.

MinIP reconstruction with phase-multiplication techniques (Reichenbach et al., 1997). The right side of Fig. 5b shows a surface rendering of the vessels in the MinIP slab, generated by manual segmenting the vessels in consecutive coronal slices. This approach gives a clearer delineation of the vessels, especially in visualizing the fine vessel branches deep in the cortical tissue.

\section{Discussion}

This work describes techniques for in vivo imaging of the intracortical vasculature at very high resolution, and with the specificity to distinguish both arterial and venous structures. These methods can be used in a variety of research applications that require knowledge of the vascular anatomy. One such application is fMRI, in which neuronal activation produces a hemodynamic response that can be detected with MRI. The methods presented here may help studies of nuerovascular coupling by providing in vivo, three-dimensional information about the vascular structure that can be used for modeling the vascular response. Previous modeling studies have been used either ex vivo data or in vivo imaging of surface vessels only (Harrison et al., 2002; Turner, 2002). This technique may also benefit studies of stroke, cancer angiogenesis, and neurovascular development.

All these applications would benefit greatly from an accurate model of the vascular network, which could be generated using the in vivo techniques described in this work. While the MR images show the position and overall architecture of the vessels quite well, they do not give the actual vessel diameters, which is critical for vascular modeling. In the flow-weighted images, the bright signals are intravascular and represent the volume of inflowing blood. 

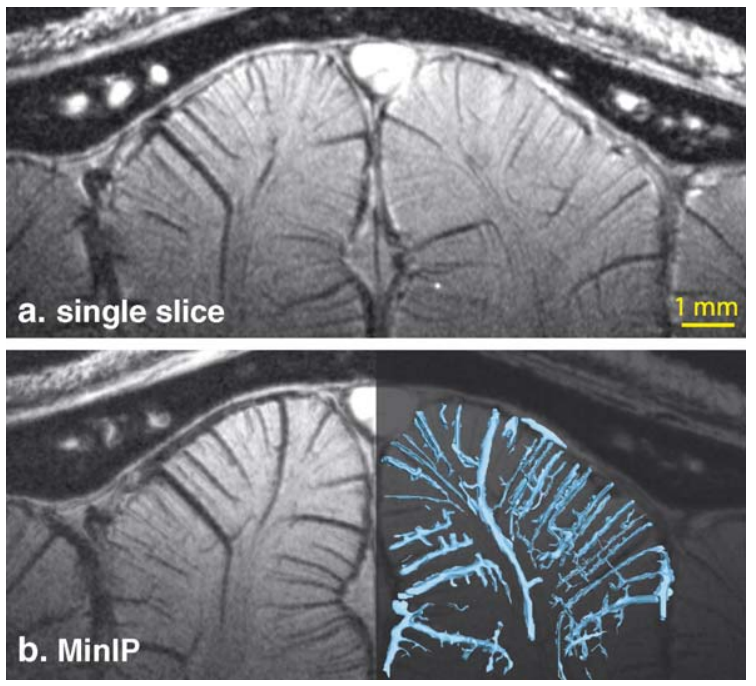

Fig. 5. Reconstructions from a high-resolution post-MION acquisition oriented in the coronal plane. The acquisition parameters include TR/TE $=$ $50 / 3.5 \mathrm{~ms}$, readout band width $20 \mathrm{kHz}$, asymmetric echo in the readout direction $(20 / 80 \%)$, FOV $20 \times 11.2 \times 2.5 \mathrm{~mm}$, matrix $384 \times 216 \times 16,16$ averages, acquisition time $2: 53 \mathrm{~min}$ per scan, total time $46: 08 \mathrm{~min}$. The image was reconstructed as in Fig. 1, with a $20 \%$ Tukey window and $2 \times$ zero-filling, producing a reconstructed resolution of $26 \times 26 \times 78 \mu \mathrm{m}$. Additionally, a 3D Gaussian filter was applied in image space for improved vessel visibility. (a) Single-slice image showing numerous penetrating and deep-branching vessels. (b) A minimum intensity projection produced over a $515-\mu \mathrm{m}$ slab shows greater vessel density than the single slice. The right side of panel $b$ shows a surface rendering of vessels from the same slab.

While flow-related artifacts can cause cancellation of some inflowing signal, it may be possible to use the bright vessel signal as a direct estimate of the size of a vessel lumen. However, the dark appearance of veins in all images and both arteries and veins in the post-MION images are caused by dephasing of extravascular signal due to susceptibility effects. Thus, the dark regions do not represent the true vessel lumen size; rather they represent the extent of extravascular $\mathrm{B}_{0}$ distortion. Therefore, the bright signals in the flow-weighted images approximate the size of the true vessel lumens, whereas the dark signals in both flow-weighted and contrast-enhanced images represent an upper limit on the vessel lumens. It would be possible to model the extent of the extravascular signal loss if the vessel geometry, blood oxygenation, and contrast agent concentration were known. Alternatively, the intra- and extravascular portions of the signal voids could be mapped by comparing these gradient-echo images to a spin echo image with a longer TE, in which the extravascular susceptibility effects would be substantially suppressed (Ogawa et al., 1993; Boxerman et al., 1995; Fujita, 2001). One or more of these approaches would need to be used to generate an accurate geometric model of the vasculature suitable for quantitative flow modeling.

The key elements to the imaging techniques presented here are the use of very high-resolution imaging, time-of-flight angiography, and susceptibility-based contrast due to exogenous agents and BOLD. The high-resolution imaging is made possible by the use of a small, sensitive surface coil, and by the inherent sensitivity of a high magnetic field. The high field also enhances susceptibility effects, giving high contrast between the cortex and deoxyhemoglobin-containing vessels even at short echo times. Additionally, the longer $T_{1}$ values associated with the high field may improve the time-of-flight image contrast by improving suppression o background tissue signal (Al-Kwifi et al., 2002; Willinek et al., 2003).

The particular application shown here, a study of the vasculature in the cat visual cortex, is especially well-suited to these techniques due to the relatively mild cortical curvature and the orderly organization of the intracortical vasculature within the imaged volume. With some adaptations, these methods can be extended to other tissues and applications. In order to acquire highresolution data in a reasonable period of time, it is necessary to have a small field-of-view (FOV). A $20 \times 20 \times 5 \mathrm{~mm}$ FOV was possible in this case by using a narrow slice-selective excitation and by taking advantage of the curvature of the posterior skull around the visual cortex. Limited FOV imaging can also be accomplished more generally by using small RF coils, outervolume suppression, or spin echo imaging with multidimensional slice selection.

Inside the cat visual cortex, most of the large vessels run perpendicular to the cortical surface and are thus approximately perpendicular to both an axial slice and the external $\mathrm{B}_{0}$ field. This enables the measurement of flow in many of the vessels with a single acquisition. If the vasculature did not have a preferred orientation, multiple acquisitions would be required to see time-offlight effects in different orientations. Additionally, because the flow effect only extends a short distance into the slab, the full extent of the arteries is not visible in the pre-MION images. More extensive flow effects could be seen by using a series of smaller slabs and combining the images, as in the Multiple Overlapping Thin-Slab Acquisition (MOTSA) technique (Blatter et al., 1991).

Note that the extent of the flow weighting was sensitive to the placement of the excitation slab. It was found that the maximum inflow effect occurred when the edge of the excitation slab was positioned in the middle of the sagittal sinus; in cases where the slab was offset more superior or inferior to this optimal location, the inflow effects were reduced but still clearly present in all animals. Reproducing this inflow effect required some care in determining the proper flip angle and slab position. The postMION effects, in contrast, were consistently observed in all animals.

The orientation of the intracortical vessels also makes susceptibility-based contrast more effective. This orientation effect can be accounted for in modeling the susceptibility artifact, so vessel size determination would still be feasible in tortuous vessels. The sensitivity for detecting small vessels, however, is determined largely by the extravascular dephasing, and will therefore be lower for vessels more closely aligned with the $\mathrm{B}_{0}$ field. The extravascular dephasing is what gives the post-MION images high sensitivity for detecting small vessels. This sensitivity can be increased by lengthening the echo time TE, but in doing so the artifacts increase in size and can overlap, reducing the visibility of individual vessels. In this work, we chose the shortest possible TE to minimize the artifact size because with a high field and a strongly paramagnetic contrast agent there is sufficient dephasing even at short echo times. Other contrast agents can be used to produce a similar effect. Whereas MION does have a high $\mathrm{T}_{1}$ relaxivity, the $\mathrm{T}_{2}^{*}$ relaxivity (i.e., susceptibility-induced dephasing) dominates when used at high doses and at high field. We performed similar studies using the more physiologically tolerable contrast agent Gd-DTPA, which has a smaller $\mathrm{T}_{2}^{*}$ relaxivity compared to MION. The post-Gd images showed similar but reduced dephasing effects. Some increased signal could be seen in arteries in the post-Gd images, presumably 
due to intravascular $T_{1}$ decrease, but in general this effect was dominated by $\mathrm{T}_{2}^{*}$ relaxation even at these short echo times. It may be feasible to perform similar studies using Gd-DTPA or another Gdcontaining contrast agent instead of MION. Gd-DTPA is not an optimal agent for these studies because its intravascular lifetime is shorter that MION and its $\mathrm{T}_{2}^{*}$ relaxivity is substantially shorter. These factors can be compensated by increasing the echo time and lowering the resolution or scanning faster, or by selecting other Gdcontaining contrast agents with different physical properties.

The post-MION tissue-weighted images have the highest sensitivity for detecting small vessels. Small arteries with undetectable flow effects, and veins too small to be seen on the pre-MION images can be seen in the post-MION images because the large susceptibility difference imparted by the MION produces dephasing of extravascular spins within a volume larger than the vessel itself. The specificity for distinguishing between veins and arteries comes primarily from the difference in the concentration of deoxyhemoglobin, and secondarily from differences in flow effects. Veins appear dark in all pre-MION images, whereas arteries are bright in the flow-weighted images and indistinguishable from the cortex in the tissue-weighted pre-MION images. Although generally applicable, there may be situations where these assumptions fail. For example, if a vein had blood flowing into the slab and had unusually low deoxyhemoglobin concentration, due to a local vascular abnormality or systemically elevated venous oxygenation, it may be misidentified as an artery.

The ability to image small vessels is limited by both image contrast and resolution. The smallest of the penetrating vessels, that is, those oriented perpendicular to the cortical surface, produced an extravascular dephasing effect with a diameter of $83 \mu \mathrm{m}$. The vessel lumen is therefore smaller, but how much smaller is not known without ex vivo measurement of vessel sizes. Note that it is possible to visualize vessels substantially smaller than the nominal image resolution because the extravascular dephasing produced by these vessels is larger then the vessels themselves, and thus the visualization is indirect.

For most of the acquisitions in this study, we used anisotropic imaging voxels with the maximum resolution in the plane perpendicular to the vessel axes. For volumetric reconstruction purposes, it would be preferable to have isotropic voxels, but this would require a decrease in resolution or an increase in scan time. Longer scan times are problematic because they increase the likelihood of animal motion. With proper animal preparation and physiological monitoring, we generally did not observe motion artifacts at these resolutions. For higher resolution studies, or studies with longer scan times, some kind of prospective or retrospective motion correction may be required.

\section{Conclusion}

This work describes a methodology for visualizing small vessels within the cortex of the cat brain. The use of very highresolution MR imaging with a combination of contrasts from blood flow, BOLD, and an exogenous iron-oxide contrast agent gives high sensitivity for visualizing small vessels and the specificity necessary to distinguish between arteries and veins with orientations perpendicular to the pial surface. When combined with modern image analysis techniques, this methodology enables researchers to visualize the cortical neurovasculature with microscopic-scale resolution.

\section{Acknowledgments}

This work was supported by the NIH grants R01MH070800, R21EB004460, and R01CA092004; the NIH (NCRR) grant P41RR008079; and the MIND Institute. Instrument acquisition was in part funded by the Keck Foundation.

This work was carried out in part using computing resources at the University of Minnesota Supercomputing Institute.

\section{References}

Al-Kwifi, O., Emery, D.J., Wilman, A.H., 2002. Vessel contrast at three Tesla in time-of-flight magnetic resonance angiography of the intracranial and carotid arteries. Magn. Reson. Imaging 20, $181-187$.

Beckmann, N., 2000. High resolution magnetic resonance angiography non-invasively reveals mouse strain differences in the cerebrovascular anatomy in vivo. Magn. Reson. Med. 44, 252.

Beckmann, N., Stirnimann, R., Bochelen, D., 1999. High-resolution magnetic resonance angiography of the mouse brain: application to murine focal cerebral ischemia models. J. Magn. Reson. 140, 442.

Besselmann, M., Liu, M., Diedenhofen, M., Franke, C., Hoehn, M., 2001. MR angiographic investigation of transient focal cerebral ischemia in rat. NMR Biomed. 14, 289.

Blatter, D.D., Parker, D.L., Robison, R.O., 1991. Cerebral MR angiography with multiple overlapping thin slab acquisition: Part I. Quantitative analysis of vessel visibility. Radiology 179, 805.

Boxerman, J.L., Hamberg, L.M., Rosen, B.R., Weisskoff, R.M., 1995. MR contrast due to intravascular magnetic susceptibility perturbations. Magn. Reson. Med. 34, 555-566.

Dobre, M.C., Marjanska, M., Ugurbil, K., 2005. Blood T 1 measurements at high magnetic field strengths. Proceedings of the 13th annual ISMRM, Miami, pp. 1162.

Duvernoy, H.M., Delon, S., Vannson, J.L., 1981. Cortical blood vessels of the human brain. Brain Res. Bull. 7, 519.

Fujita, N., 2001. Extravascular contribution of blood oxygenation leveldependent signal changes: a numerical analysis based on a vascular network model. Magn. Reson. Med. 46, 723-734.

Gruetter, R., 1993. Automatic, localized in vivo adjustment of all first- and second-order shim coils. Magn. Reson. Med. 29, 804

Harel, N., Lin, J., Moeller, S., Ugurbil, K., Yacoub, E., 2006. Combined imaging-histological study of cortical laminar specificity of fMRI signals. NeuroImage 29 (3), 879-887.

Harris, F.J., 1978. On the use of windows for harmonic analysis with the discrete Fourier transform. Proc. IEEE 66, 51.

Harrison, R.V., Harel, N., Panesar, J., Mount, R.J., 2002. Blood capillary distribution correlates with hemodynamic-based functional imaging in cerebral cortex. Cereb Cortex 12, 225-233.

Krucker, T., Schuler, A., Meyer, E.P., Staufenbiel, M., Beckmann, N., 2004. Magnetic resonance angiography and vascular corrosion casting as tools in biomedical research: application to transgenic mice modeling Alzheimer's disease. Neurol. Res. 26, 507.

Liang, Z.P., Boada, F.E., Constable, R.T., Haacke, E.M., Lauterbur, P.C., Smith, M.R., 1992. Constrained reconstruction methods in MR imaging. Rev. Magn. Reson. Med. 4, 67.

Mellin, A.F., Cofer, G.P., Smith, B.R., Suddarth, S.A., Hedlund, L.W., Johnson, G.A., 1994. Three dimensional magnetic resonance microangiography of rat neurovasculature. Magn. Reson. Med. 32, 199

Ogawa, S., Lee, T.M., Nayak, A.S., Glynn, P., 1990. Oxygenation-sensitive contrast in magnetic resonance image of rodent brain at high magnetic fields. Magn. Reson. Med. 14, 68.

Ogawa, S., Menon, R.S., Tank, D.W., Kim, S.G., Merkle, H., Ellermann, J.M., Ugurbil, K., 1993. Functional brain mapping by blood oxygenation level-dependent contrast magnetic resonance imaging. Biophys. J. 64,800 . 
Park, S.H., Hayashi, T., Kim, S.G., 2005. Determination of intracortical venous vessel density using venography at $9.4 \mathrm{~T}$. Proceedings of the 13th annual ISMRM, Miami, p. 1410.

Pfeuffer, J., Juchem, C., Merkle, H., Nauerth, A., Logothetis, N.K., 2004. High-field localized 1H NMR spectroscopy in the anesthetized and in the awake monkey. Magn. Reson. Imaging 22, 1361.

Reese, T., Bochelen, D., Sauter, A., Beckmann, N., Rudin, M., 1999. Magnetic resonance angiography of the rat cerebrovascular system without the use of contrast agents. NMR Biomed. 12, 189.

Reichenbach, J.R., Venkatesan, R., Schillinger, D.J., Kido, D.K., Haacke, E.M., 1997. Small vessels in the human brain: MR venography with deoxyhemoglobin as an intrinsic contrast agent. Radiology 204, 272.

Shen, T., Weissleder, R., Papisov, M., Bogdanov Jr., A., Brady, T.J., 1993. Monocrystalline iron oxide nanocompounds (MION): physicochemical properties. Magn. Reson. Med. 29, 599-604.

Turner, R., 2002. How much cortex can a vein drain? Downstream dilution of activation-related cerebral blood oxygenation changes. NeuroImage $16,1062-1067$.

Willinek, W.A., Born, M., Simon, B., Tschampa, H.J., Krautmacher, C., Gieseke, J., Urbach, H., Textor, H.J., Schild, H.H., 2003. Time-of-flight MR angiography: comparison of 3.0-T imaging and 1.5-T imaginginitial experience. Radiology 229, 913-920. 